\section{CALCULATION OF SPINDLE COMPLIANCE CONSIDERINGS IT'S INTERACTION WITH MACHINE FRAME}

M. Sulitka, P. Kolar

Research Center for Manufacturing Technology Faculty of Mechanical Engineering, CTU in Prague, Czech Republic

e-mail:m.sulitka@rcmt.cvut.cz

The calculation of spindle dynamic compliance is usually performed taking into account only the mechanical system of the spindle and tool, while the influence of the machine tool frame is neglected.

However, when performing machining operations characterized by high cutting forces - which typically arise e.g. during roughing - machine frame vibration may be induced. Under such conditions, a model of the spindle alone is not sufficient to predict the dynamic behaviour of the spindle; therefore, coupled models of the spindle and machine frame system need to be created. The paper presents two possible approaches that can be used for such tasks. The first approach consists in modelling the whole mechanical system in the Finite Element (FE) environment; the second one employs the technique of coupling modally reduced separate models of flexible bodies. The application of both approaches and their comparison is illustrated by an example of a real machine tool model. The influence of the machine tool frame on the spindle dynamic compliance is demonstrated using the machine tool coupled models.

Keywords

Spindle dynamic properties, Coupled models of spindle and machine frame, Modal decomposition, Modal reduction, FEM

\section{Introduction}

The main aim of machine tool design is to achieve long-term precision and high cutting productivity, which directly influence the operating costs of the machine. However, increases in productivity are very often limited by chatter occurring in the machine - tool - workpiece system. The type of cutting operation and the tool employed determine which part of the system will be excited and which will begin to vibrate with the highest amplitude.

The common approach to chatter prediction is based on calculating only the frequency transfer functions of the spindle and tooling system [Altintas 2004, Altintas 2005a]. However, as mentioned above, the behaviour of a spindle may be in some cases influenced by its interaction with the machine tool frame. Under such conditions, relevant predictions of the spindle dynamic properties can be made using a model of the whole spindle-machine frame system [Cao 2007].

The coupling of the spindle and machine frame models, possibly complemented with a tool model, also represents an important contribution to the tasks of advanced tool tip movement and workpiece surface quality simulations, performed using machine tool virtual models [Altintas 2005b], [Zaeh 2007], [Vesely 2008].

The paper describes two possible approaches to creating spindle and machine frame coupled models. In both cases the finite element (FE) modelling technique is employed. The first approach is based on modelling the whole mechanical system in the FE environment (the model created using this approach is further referred to as the "Full FE model"), whereas the second utilizes the principle of coupling separate, modally reduced FE models outside of the FE environment (further referred to as "Coupled modelling"). Simulations of the spindle dynamic compliance are performed using an example of a real machine tool model.

\section{The full FE model of the spindle and machine frame system}

\subsection{Method characteristics}

The main advantage of the spindle and machine frame Full FE model is that an uninterrupted force flow throughout the whole model is ensured. Results of the static and modal analysis are easy to display, which, in many cases, can help to visually identify critical regions of the machine frame structure. A FE model of the system is created in three principal steps:

- a FE model of the spindle,

- a FE model of the machine tool structure,

- connection of both FE models,

followed by a modal analysis, and possibly a harmonic response calculation.

The importance of full FE modelling for a comprehensive evaluation of machine tool structure design is discussed e.g. in [Smolik 2007].

\subsection{The Spindle FE model}

A motorized spindle of a German spindle producer has been considered. The spindle has three bearings FAG XCB 71920E in TBT arrangement in the front group. There is one spring preloaded bearing FAG HCS 71914E at the rear end.

A solid model of the spindle has been prepared using the CAD/ FEM software I-DEAS (Fig. 1). The FE computational mesh is created as solid quadratic for both the shaft and the spindle housing.

Bearing stiffness values are determined using a model based on the theory introduced in [Harris 1991]. This model describes the dependence of the bearing internal condition (contact angles, contact forces, contact deformation) on its external condition (external load, external deformation) [Kolar 2007], [Holkup 2006]. The bearing model is also able to take account of changes in the bearing stiffness due to the shaft rotation (Fig. 2). Catalogue data on bearing dimensions, the Hertz contact theory and the bearing inner geometry changes due to the ring curvature centre movement are considered in the calculation. The bearing model makes it possible to compute the total radial and axial stiffness of the bearing. The value of the bearing total stiffness is distributed among the spring elements created in the FE model (Fig. 1) along the spindle shaft

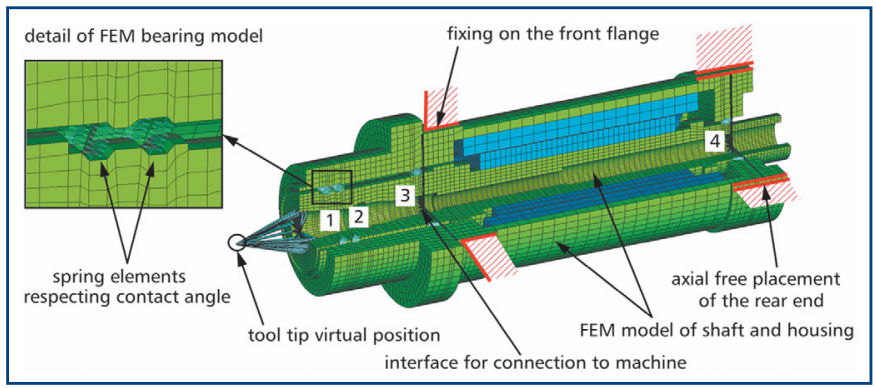

Figure 1. The spindle FE model

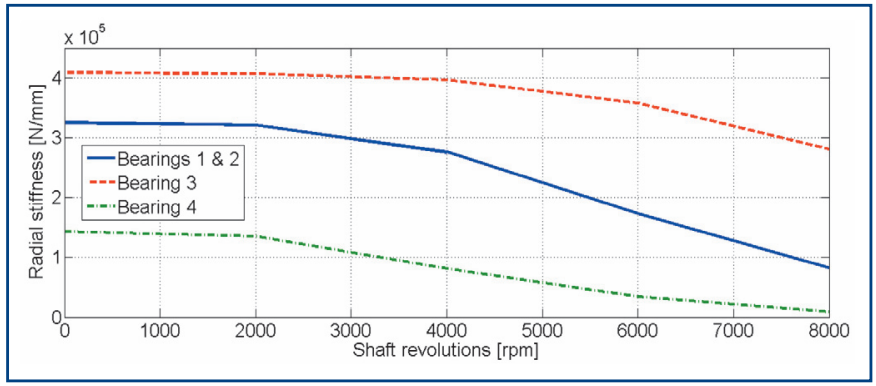

Figure 2. Radial stiffness of the spindle bearings as a function of the shaft revolutions 
circumference. Each of the spring elements is defined by its axial and radial stiffness values. Since the spring elements in the FE model are inclined according to the real contact angle of the balls in the bearing, tilting moment properties of the bearing are implicitly taken into account.

To calculate the spindle properties, a boundary condition fixing all of the DOFs at the front flange and the radial movement at the spindle rear end has been applied. The spindle FE model contains groups of auxiliary rigid elements as well. These elements provide interfaces for connecting the spindle model to the machine frame and connecting the tool tip with the spindle shaft.

\subsection{The FE Model of the machine tool structure}

The spindle mentioned above is used in a vertical bridge type milling machine. The geometrical model of the machine tool structure as well as the computational FE mesh have been prepared in the I-DEAS software (Fig. 3). All of the machine tool structural parts are modelled using a solid quadratic mesh. The mechanical structure of the ball screw feed drives is represented by the spring and beam elements.

All of the linear motion axes are equipped with a MRB 45 linear guideway. Each carriage is modelled in a simplified form by a triplet of linear spring elements arranged in parallel with the guideway direction. In the transversal direction, the spring elements feature linearized stiffness values according to the catalogue data; in the lengthwise direction there is a zero stiffness value allowing for free movement of the motion axis.

The ram model includes two groups of auxiliary rigid elements for connecting the spindle.

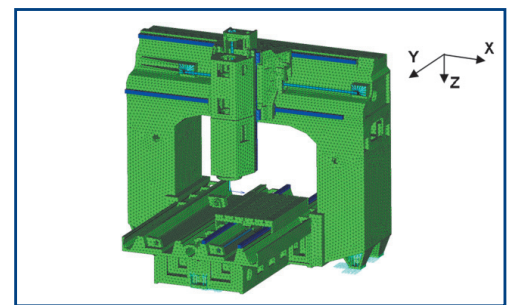

Figure 3. The FE Model of the machine frame

\subsection{The spindle and machine frame FE assembly}

The spindle and machine frame FE models are joined in two interface points mentioned in the previous chapters. A rigid joint representing a fixed mounting is established between the couple of points close to the spindle front end; the other couple of points on the spindle rear end is interconnected by a spring with radial stiffness; free axial movement is allowed according to the real design. A detailed view of the spindle and machine ram connection can be seen in Fig. 4.

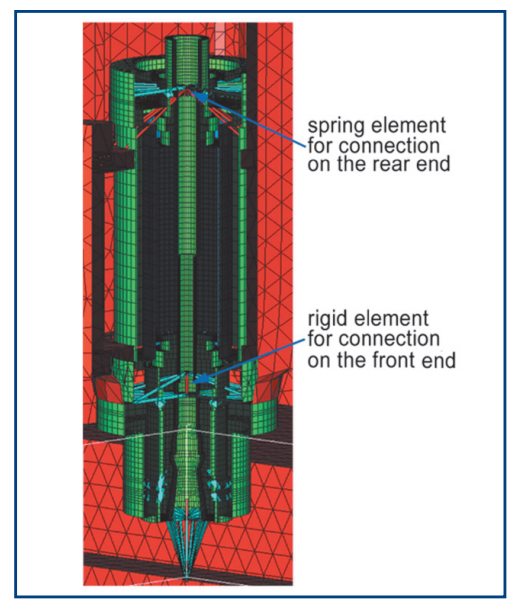

Figure 4. Detail of the connection between the spindle and ram

\subsection{Modal Analysis and Harmonic Response calculation}

The frequency response function (FRF) can be obtained from a harmonic response task in the FE environment. However, this kind of analysis is quite time consuming; therefore, the strategy of transforming the model into a the State space description and subsequently evaluating the FRF in Matlab/Simulink has been chosen. Since the FE solver does not provide a direct possibility to create the State space model, the modal decomposition technique has been employed for the transformation. During the transformation process only the nodes needed to evaluate the FRFs are selected and, at the same time, the number of exported eigenfrequencies can be reduced to the most important ones. In our case, 30 eigenfrequencies have been included. Modal damping of all the eigenfrequencies has been set constant to $2.5 \%$.

\subsection{Simulated dynamic properties of the spindle in free-free state}

At first, the modal properties of the spindle in free-free state and the FRFs at the spindle nose have been calculated for two values of the spindle revolutions. The resulting FRFs are shown in Fig. 5. In a static state the spindle features two significant eigenfrequencies. The eigenform at $1010 \mathrm{~Hz}$ is characterized by shaft bending (Fig. 6); at the frequency of $1530 \mathrm{~Hz}$ the rear end of the shaft oscillates in the rear bearing. Bearing stiffness values decrease as the shaft revolutions increase, which results in falling eigenfrequency values and elevated dynamic compliances for both of the significant eigenforms. Therefore, it is possible to say that the rear bearing stiffness and shaft geometry are parameters with the most significant influence on the FRFs evaluated at the nose of the free spindle.

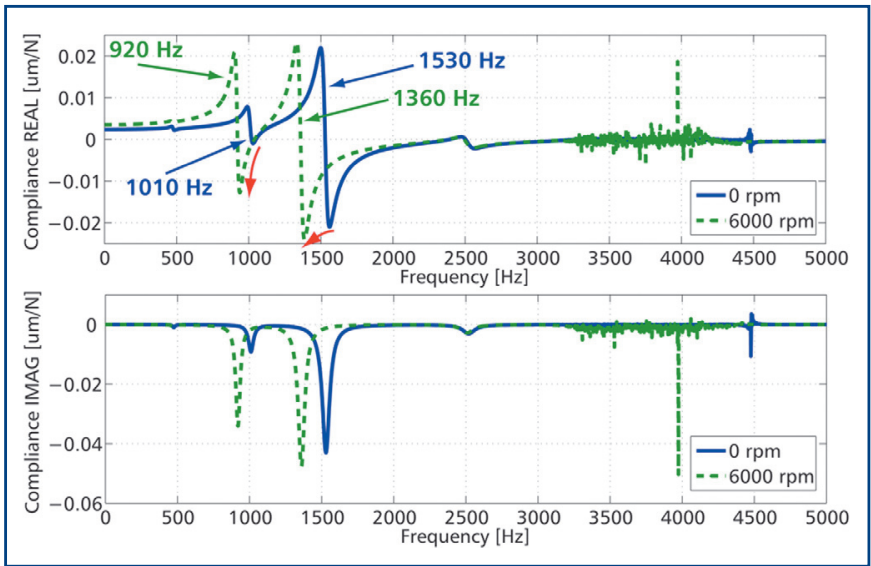

Figure 5. Simulated FRFs at the nose of the spindle in free-free state. The cases of 0 and $6000 \mathrm{rpm}$ of the spindle shaft are considered

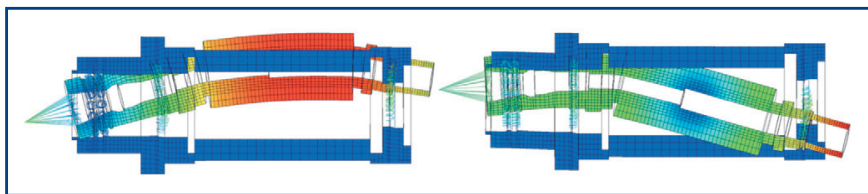

Figure 6. Important eigenforms of a spindle in free-free state at $0 \mathrm{rpm}$. The eigenform for $1010 \mathrm{~Hz}$ is shown on the left, the eigenform for $1530 \mathrm{~Hz}$ on the right.

\subsection{Simulated dynamic properties of the spindle connected} with the machine frame

The Full FE model of the spindle and machine frame structure makes it possible to observe an influence of the machine frame on the FRFs at the spindle nose. The chart shown in Fig. 7 reveals a shift in dominant dynamic compliances of the system from the range above $1000 \mathrm{~Hz}$ to the range between $50 \mathrm{~Hz}$ and $200 \mathrm{~Hz}$. A detail of the low frequency range is depicted in Fig. 8. 


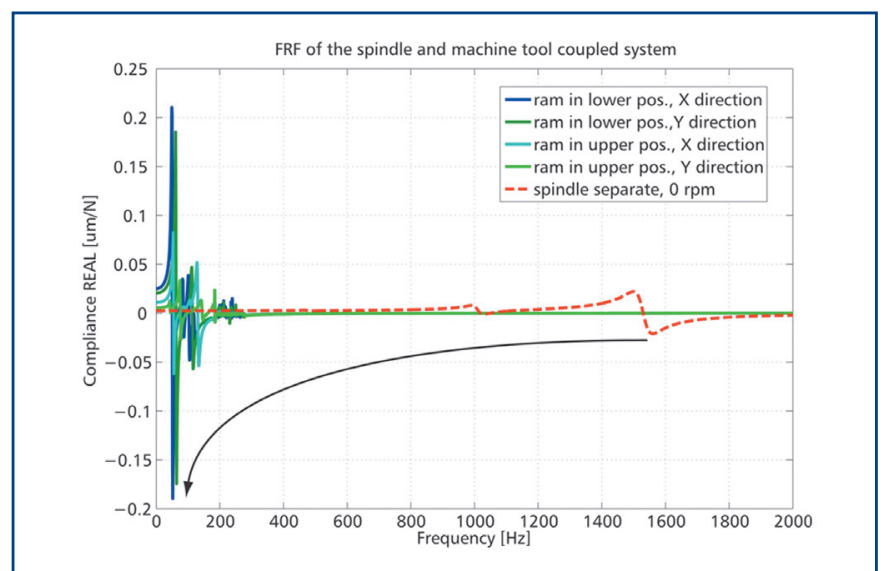

Figure 7. The shift of critical eigenfrequencies observed for the spindle model connected with the machine frame

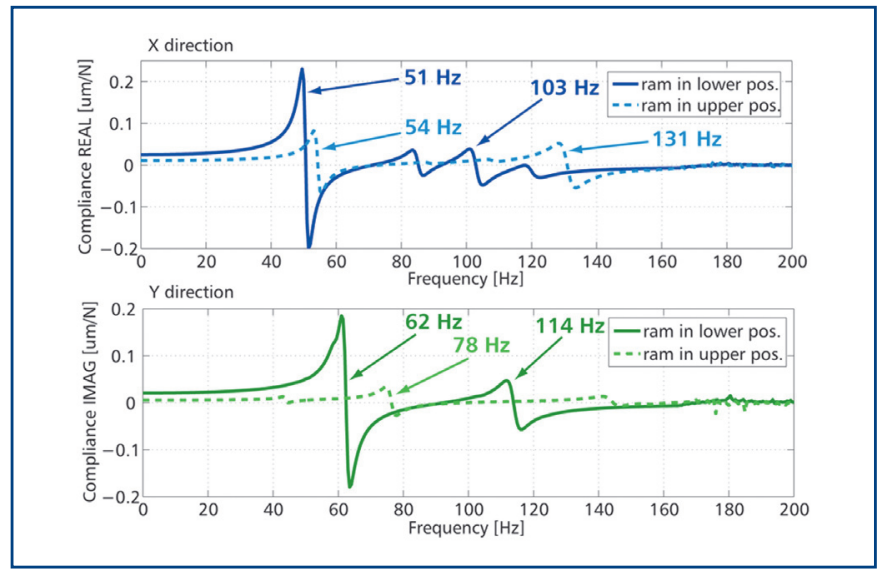

Figure 8. Detail of the simulated FRFs at the spindle nose for the model connected with the machine frame. The $X$ and $Y$ directions are presented.

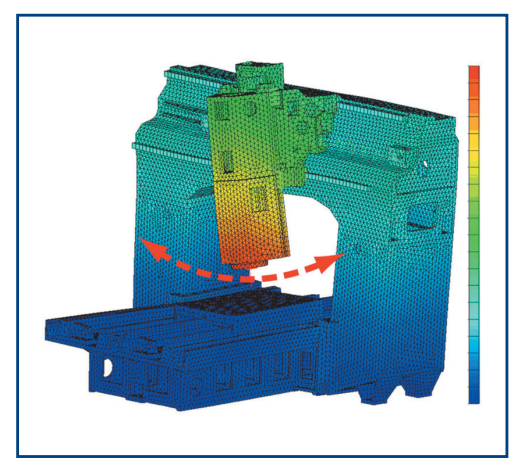

Figure 9. The machine tool structure eigenform of $51 \mathrm{~Hz}$

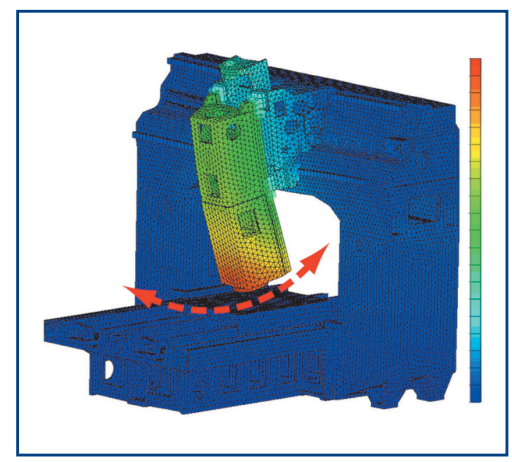

Figure 10. The machine tool structure eigenform of $62 \mathrm{~Hz}$
The maximum dynamic compliance in the $X$ direction occurs at $51 \mathrm{~Hz}$, with the ram in the lowest position. The eigenform of this eigenfrequency is characterized by the ram swinging in the $X$ direction, and the cross slide moving in the $X$ direction (Fig. 9). The frequency and dynamic compliance of this eigenform change when the ram moves to the upper position.

In the $Y$ direction the greatest dynamic compliance occurs at the frequency of $62 \mathrm{~Hz}$, with the ram in the lowest position. The corresponding eigenform is characterized by the ram bending in the $Y$ direction (Fig. 10). Similarly to the $X$ direction properties, the value of the critical frequency and dynamic compliance is affected by the ram position in the vertical direction.

The results clearly show that in the low frequency range, the spindle nose critical dynamic compliance is determined by the machine structure properties, and depends on the ram position.

\section{Coupling the separate spindle}

\section{and machine frame models}

The approach consisting in coupling the separate models of the spindle and the machine frame outside of the FE environment is an effective alternative to the strategy of creating one coupled FE model. Within the strategy employed, modally reduced models of flexible bodies are considered. The spindle and the machine tool frame are characterized by eigenfrequencies occurring in significantly different frequency ranges, which means that creating a coupled model of the system does not require a large number of eigenfrequencies describing the behaviour of each body. The applicability of various techniques used for coupling the models of elastic bodies is discussed e.g. in [Lehner 2006].

As described in Paragraph 2.4, the connection between the spindle and the machine frame model is established using spring elements in two interface points on the spindle axis. The principle of coupling both compliant bodies using the $X, Y$ a $Z$ springs is shown in Fig. 11.

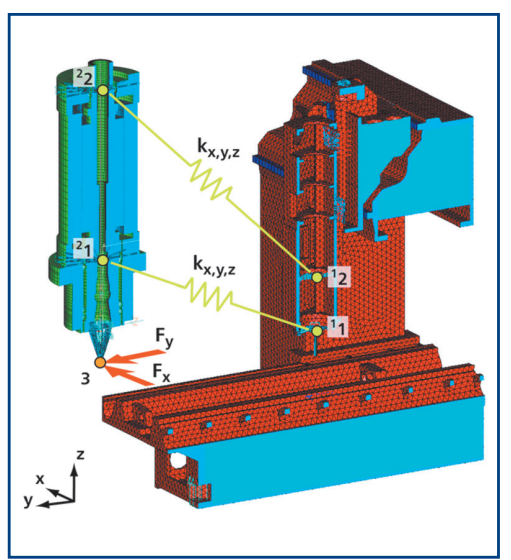

Figure 11. Coupling separate models of the spindle and the machine frame

The interface point 1 (point 1 is part of the machine ram, point ${ }^{2} 1$ is part of the spindle model) is a rigid connection of the spindle front flange with the machine tool ram. Therefore, the stiffness of the coupling spring in this point is set to high values of $\left(\left\{1.10^{12}, 1.10^{12}\right.\right.$, $\left.\left.1.10^{12}\right\}\right) \mathrm{N} / \mathrm{m}$ in the $X, Y$ and $Z$ direction. The rear end of the spindle is fixed in the ram only in the radial direction; therefore, the coupling spring between the interface points ${ }^{12}$ and ${ }^{2} 2$ is defined by the stiffness values of $\left(\left\{1.10^{12}, 1.10^{12}, 0\right\}\right) \mathrm{N} / \mathrm{m}$. Fig. 11 also illustrates the $F_{x}$ and $F_{Y}$ excitation forces at the tool tip which are used for evaluating the $X$ and $Y$ directional FRFs.

Both the spindle model and the machine frame model are subjected to modal analysis, based on which the transformation into a State space description is performed. The linear, time-invariant state space model in modal coordinates is written as 


$$
\begin{aligned}
& \dot{\mathbf{q}}_{S}=\mathbf{A} \cdot \mathbf{q}_{S}+\mathbf{B} \cdot \mathbf{u} \\
& \mathbf{y}=\mathbf{C} \cdot \mathbf{q}_{S}
\end{aligned}
$$

with $\mathbf{u}$ as the input vector, $\boldsymbol{y}$ as the output vector and the state vector defined in the modal coordinates by $\mathbf{q}_{S}=\left[\begin{array}{ll}\dot{\mathbf{q}} & \mathbf{q}\end{array}\right]^{T}$. State space matrices are defined as

$$
\mathbf{A}=\left[\begin{array}{cc}
\mathbf{0} & \mathbf{E} \\
-\boldsymbol{\Lambda} & -\mathbf{C}_{q}
\end{array}\right], \quad \mathbf{B}=\left[\begin{array}{c}
0 \\
\mathbf{V}^{T}
\end{array}\right], \quad \mathbf{C}=\left[\begin{array}{ll}
\mathbf{V} & \mathbf{0}
\end{array}\right]
$$

where $\boldsymbol{\Lambda}$ is the spectral matrix of eigenfrequencies, $\mathbf{C q}$ the diagonal matrix of modal damping and $\mathbf{V}$ the modal matrix of eigenvectors.

The input equation of the State space coupled system is formulated by joining the equations of two separate bodies

$$
\left[\begin{array}{c}
{ }^{1} \dot{\mathbf{q}}_{S} \\
{ }^{2} \dot{\mathbf{q}}_{S}
\end{array}\right]=\left[\begin{array}{cc}
{ }^{1} \mathbf{A} & 0 \\
0 & { }^{2} \mathbf{A}
\end{array}\right] \cdot\left[\cdot\left[\begin{array}{c}
{ }^{1} \mathbf{q}_{S} \\
{ }^{2} \mathbf{q}_{S}
\end{array}\right]+\left[\begin{array}{cc}
{ }^{1} \mathbf{B} & 0 \\
0 & { }^{2} \mathbf{B}
\end{array}\right] \cdot\left[\begin{array}{c}
{ }^{1} \mathbf{u} \\
{ }^{2} \mathbf{u}
\end{array}\right],\right.
$$

where the superscript 1 denotes body 1 (machine frame) and the superscript 2, body 2 (spindle).

Mutual force interaction between the bodies is defined as

$$
-{ }^{1} u_{i}={ }^{2} u_{i}=k_{i} \cdot\left({ }^{1} x_{i}-{ }^{2} x_{i}\right)
$$

After the vector of linear displacements $x=V . q$ is expressed as a linear combination of the shape matrix $\mathbf{V}$ and the modal coordinates $\mathbf{q}$, after substitution of (4) into (3) the input equation is obtained in the following matrix form:

$$
\left[\begin{array}{c}
{ }^{1} \dot{\mathbf{q}}_{S} \\
{ }^{2} \dot{\mathbf{q}}_{S}
\end{array}\right]=\left[\begin{array}{ll}
\mathbf{A}_{C 1} & \mathbf{A}_{C 2} \\
\mathbf{A}_{C 3} & \mathbf{A}_{C 4}
\end{array}\right] \cdot\left[\begin{array}{c}
{ }^{1} \mathbf{q}_{S} \\
{ }^{2} \mathbf{q}_{S}
\end{array}\right]+\left[\begin{array}{cc}
{ }^{1} \mathbf{B}_{r} & 0 \\
0 & { }^{2} \mathbf{B}_{r}
\end{array}\right] \cdot\left[\begin{array}{c}
{ }^{1} \mathbf{u}_{r} \\
{ }^{2} \mathbf{u}_{r}
\end{array}\right],
$$

in which the matrix $\boldsymbol{A}$ is filled with new elements and the matrix $\boldsymbol{B}$ and the vector $u$ contain only the DOFs of the nodes $r$, used as external inputs of the coupled system. In the case of the spindle and machine frame model, the system force input is defined on the spindle nose. The model created by means of the above-mentioned strategy is referred to as the Coupled model.

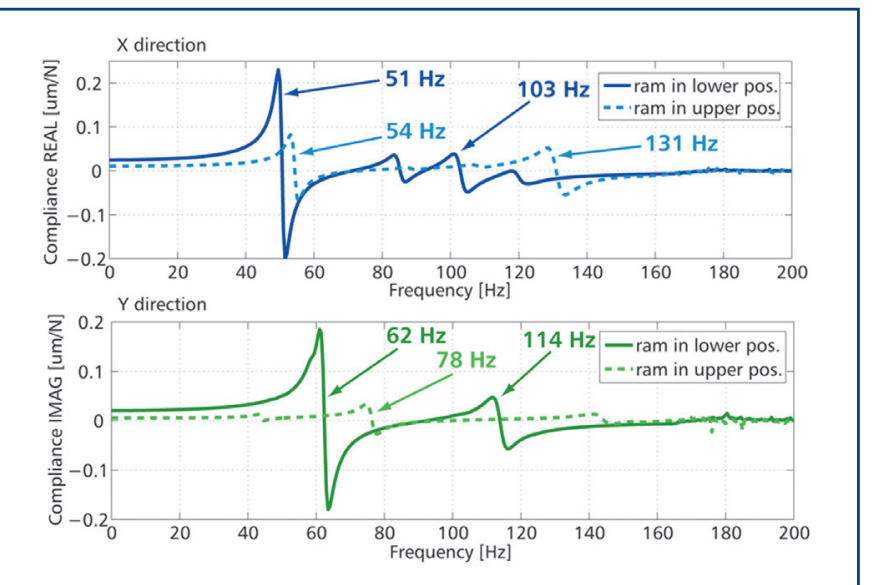

Figure 12. Dynamic compliance at the tool tip. Comparison of the Full FE model and the Coupled model.

Fig. 12 shows a comparison of FRF evaluated at the spindle nose in the $X$ and $Y$ direction by means of the Full FEM model and the Coupled model. Both the spindle and machine frame models cover their first 30 eigenfrequencies. It can be seen that all of the critical eigenfrequencies and compliances calculated by the Coupled model are in very good agreement with the Full FE model.

The shift in the critical compliance evaluated at the ram front end, both without and with a spindle, is shown in Fig. 13. In the $X$ direction the frequency of the critical compliance shifts from approx. $60 \mathrm{~Hz}$ to $51 \mathrm{~Hz}$, with the ram in the lower position; in the $Y$ direction the frequency of the critical compliance shifts from $80 \mathrm{~Hz}$ to $62 \mathrm{~Hz}$.

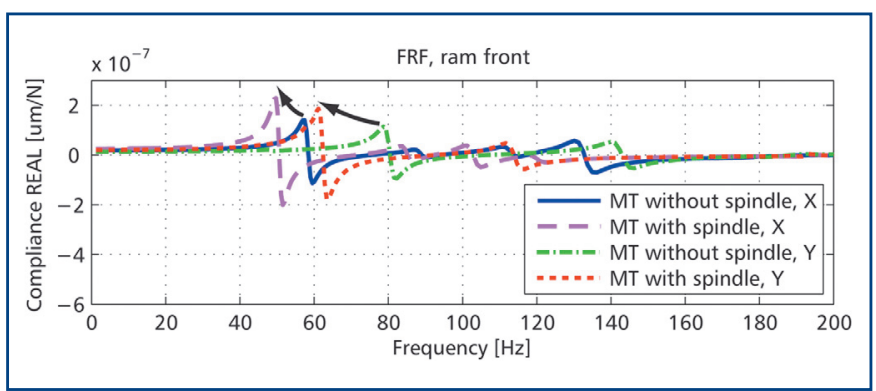

Figure 1. The spindle FE model

\section{Conclusion}

The paper analyses the influence of the machine frame on the dynamic properties evaluated at the spindle nose. Using an example of a real vertical bridge type milling machine tool, it has been demonstrated that the dominant dynamic compliance evaluated at the spindle nose is determined by the machine frame properties.

Two possible strategies for creating coupled models have been considered. One of them is the Full FE model of the whole system. The advantage of this approach is that it can provide an overall visual representation of the static and modal deformations of the machine structure. However, this kind of modelling is connected with higher computational time demands and lower flexibility when investigating the influence of different parameters of the model.

An alternative to the Full FE modelling approach is a flexible multi-body strategy, whereby models of single bodies are coupled outside the FE-environment. An approach based on the transformation of the FE models into a State space description employing the modal decomposition technique has been used. It has been shown that, for the type of machine tool structure considered, this technique can provide a relevant approximation of the dynamic properties even with a relatively small number of eigenfrequencies included in the model of each flexible body.

The modelling of the spindle and machine frame coupled systems is also related to the prediction of stable machining conditions. Especially in roughing machining operations high cutting forces arise, which can induce significant machine frame vibration. Models using the flexible multi-body strategy can be complemented by detailed models of various tools, as well as kinematical models of ball screw feed drives and their control, the influence of which on the machining process stability is studied e.g. in [Soucek 2008]. The modularity of the coupled models represents a very effective tool in machine tool development, whereby various factors influencing the FRFs at the tool tip can be investigated.

\section{Acknowledgements}

This research has been supported by the 1 M0507 grant of the Ministry of Education of the Czech Republic.

\section{References}

[Altintas 2004] Altintas, Y., Weck, M. Chatter stability of metal cutting and grinding. Annals of CIRP Vol. 53, Issue 2, 2004, p. 619-642.

[Altintas 2005a] Altintas, Y., Cao, Y. Virtual Design and Optimization of Machine Tool Spindles. Annals of CIRP, Vol. 54, Issue 1, 2005, p. 379-382.

[Altintas 2005b] Altintas, Y., Brecher, C., Weck, M., Witt, S. Virtual Machine Tool. Annals of CIRP, Vol. 54, Issue 2, 2005, p. 115-138. [Cao 2007] Cao, Y., Altintas, Y. Modeling of spindle-bearing and machine tool systems for virtual simulation of milling operations. International Journal of Machine Tools and Manufacture, Vol. 47, Issue 9, July 2007, p. 1342-1350. 
[Harris 1991] Harris, T. A. Rolling Bearings Analysis. Third Edition. New York: John Wiley \& Sons 1991.

[Holkup 2006] Holkup, T., Holy, S. Complex modelling of spindle rolling bearings. Journal of Machine Engineering

- Eficiency Development of Manufacturing Machines, Vol. 6, No.3, 2006, p. 48-61.

[Kolar 2007] Kolar, P., Holkup, T. Modeling of a Machine Tools Spindle using a Hybrid Model. In: Proc. 3rd International Conference - Virtual Design and Automation. Poznań, 2007, CD-ROM, paper No. S05 0601 kolar. 8 p.

[Lehner 2006] Lehner, M., Eberhard, P. Modellreduktion in elastischen Mehrkörpersystemen. Automatisierungstechnik 54 (2006), Oldenbourg Wissenschaftsverlag 2006

[Smolik 2007] Smolik, J. Machine tool structural parts made from unconventional materials. Ph.D. Thesis. VCSVTT,

CTU in Prague, 2007

[Soucek 2008] Soucek, P. Feed drive influence on machining process stability. In: Proc. International Congress Matar Praha 2008
- Proceedings of Part 2: Testing, Technology. Prague, Society for machine tools, 2008, p. 75 - 80

[Vesely 2008] Vesely, J., Sulitka, M. Machine Tool Virtual Model. In: International Congress Matar Praha 2008

- Proceedings of Part 1: Drives \& Control, Design, Models \& Simulation. Prague, Society for machine tools, 2008, p. $115-122$.

[Zaeh 2007] Zaeh, M. F., Hennaver, M., Poehler, A. The Machine Tool of Tomorrow: An Optimised, Complete System.

In: 6th International Conference on High Speed Machining, 21-22 March 2007, San Sebastián, Spain

\section{Contacts:}

Ing. Matej Sulitka, Ph.D.

Research Center for Manufacturing Technology,

Faculty of Mechanical Engineering,

CTU in Prague, Czech Republic

Horska 3, 12800 Praha 2, Czech Republic

tel.: +420221990 944,e-mail: m.sulitka@rcmt.cvut.cz 